\title{
INTELLIGENT OPTICAL SENSORS USING ARTIFICIAL NEURAL NETWORK APPROACH
}

\author{
Ireneu Dias, Rui Oliveira, Orlando Frazão \\ INESC Porto, Rua do Campo Alegre, 687, 4169-007 Porto, Portugal. \\ INEGI,, Rua do Barroco, 174, 4465-591 Leça do Balio, Portugal. \\ idias@inescporto.pt,ofrazao@inescporto.pt,roliveira@inegi.pt
}

\begin{abstract}
This work present and demonstrated an applications of artificial neural network approach in optical sensing. The conventional matrix method used in simultaneous measurement of strain and temperature with optical Bragg gratings is compared with artificial neural network approach. The alternative method is proposed for reduced the error.
\end{abstract}

\section{INTRODUCTION}

According to (Culshaw, 1996), a smart structure can be defined as one that monitors itself and/or its environment in order to respond to changes in its condition. Fibre Bragg grating sensors (FBG) can be very useful in applications where layered materials, such as composites, are involved. Due to the fact that fibre optic sensors are small, multiplexable, electrically isolated and immune to electromagnetic fields, they can give engineers the possibility to incorporate a fibre optic nervous systems into their composite material designs. These sensors allow measurement of parameters such as load/strain, vibration, temperature and detection of cracks and delamination phenomena (Culshaw, 1996). When FBGs are applied to the measurement of strain their cross-sensitivity to temperature is an issue that needs to be addressed.

One approach to solve this problem it is to design sensing heads insensitive to temperature. Another one is the conception of structures with sufficient degrees of freedom to permit the simultaneous discrimination of these two parameters. However, when the sensing head contains two devices, the error increases due to the cross component of the physical parameter. Usually, the matrix method to solve the problem is acceptable, but when the instability of the matrix is high, it is necessary to apply other alternative solutions. Other disadvantage of the matrix method is when the response of the sensors is non linear. This problem appears when the sensors are characterized in temperature or embedded in laminated composites.

In this work the authors present an alternative solution using an artificial neural approach to reduce the errors obtained by the matrix method in optical fibre Bragg grating embedded in laminated composite. The optical sensor presents linear response for strain and non linear response for temperature. 


\section{ARTIFICIAL NEURAL NETWORK APPROACH}

An artificial neural network (ANN) approach is proposed to improve the strain and temperature measurements. ANN has been applied to temperature and strain measurements in the case of large cross-sensitivity sensors, whose matrix inversion causes significant errors due to the nonlinear evolution of the matrix coefficients as function of $\Delta T$ and $\Delta \varepsilon$

To avoid the matrix inversion, an ANN was trained to perform a nonlinear inputoutput mapping. The variations $\Delta \lambda_{1}$ and $\Delta \lambda_{2}$ were given as inputs to the ANN which provides values of $\Delta T$ and $\Delta \varepsilon$ as outputs.

An ANN is an interconnected group of simple processing units (neurons) that uses a mathematical or computational model for information processing based on a connectionist approach to computation (Fig. 1). Despite the simplicity of each processing unit, the use of many neurons guarantees the execution of multiple tasks.

The links between neurons are characterized by weights, $w_{k i}$, which modulate the effect of the associated input, $x_{i}$, to a neuron, $k$. A pondered sum of the weighted input is then performed. The neuron transmits an activity level transduced by a function of activation, $\varphi$.

ANN is an adaptive model that can learn from the data and generalize it. It extracts the essential characteristics from the numerical data. This offers a convenient way to create an implicit model.

The chosen network was a multilayer perceptron (MLP) trained in supervised learning with the Levenberg-Marquardt algorithm. The MLP is based on an input, a hidden and an output layers interconnected in a feed-forward way (Haykin, 1999). Each neuron has directed connections to all the neurons of the subsequent layer (Fig. 2). The neurons activation function in the hidden layer is the non-linear sigmoid function whereas in the output layer it is the linear function. The network output is thus a linear combination of the outputs of the hidden neurons with $\alpha_{j k}$ defining the synaptic weights of output layer.

The MLP outputs are compared to the pretended predefined target using an errorfunction. This error is then fed back through the network. Using this information, the algorithm adjusts the weights of each connection in order to reduce the error value. The process is repeated until the network converges to some state where the error is small. In this case the network has learned a certain target function.

The universal approximation theorem for ANN states that every continuous function that maps intervals of real numbers to some output interval of real numbers can be approximated arbitrarily closely by a MLP with just one hidden layer (Bishop, 1995). $\Delta T$ and $\Delta \varepsilon$ can thus be approximated as follows:

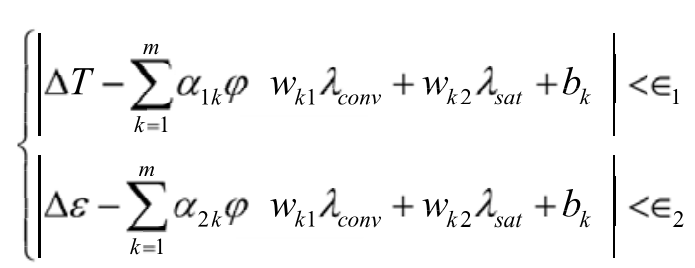

where $\varphi$ is the sigmoid function, $b_{k}$ the bias, the approximation errors and $m$ the number of neurons in the hidden layer. 


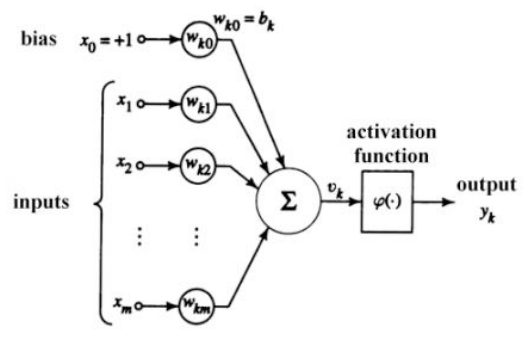

Fig. 1. Model of a neuron.

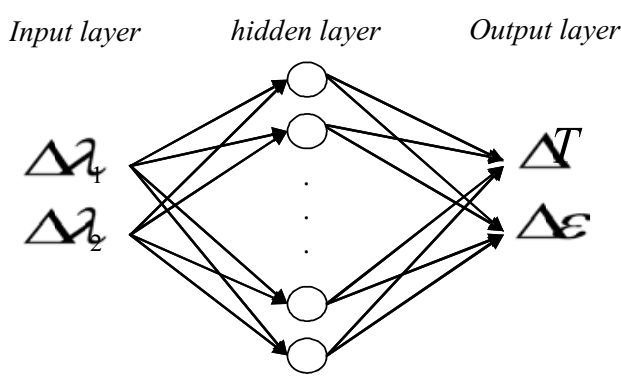

Fig. 2. Architectural graph of a MLP with one hidden layer.

The hidden layer of the chosen MLP is constituted of 10 neurons. Classical backpropagation training algorithm is based on the gradient descent method. This method is often too slow for practical problems. The Levenberg-Marquardt back-propagation algorithm is then used here instead of MLP training because of having a faster convergence (Bishop, 1995). The performance of the trained ANN is measured using the mean square error.

Data analysis was performed using MATLAB ${ }^{\circledR}$ Neural Network Toolbox (Demuth and Beale). The network inputs consist of pairs of $\left(\Delta \lambda_{1}, \Delta \lambda_{2}\right)$. During the training the MLP is adjusted to relate these input pairs to their respective target pair $(\Delta T, \Delta \varepsilon)$.

The training efficiency highly depends on the data used for training. This data must be representative of the underlying model. Training data consisted of 3400 pairs of $(\Delta T, \Delta \varepsilon)$ values generated, within a range of $0-2700 \mu \varepsilon$ and $0-45{ }^{\circ} \mathrm{C}$, by interpolation of the experimental values. The respective pairs of $\left(\Delta \lambda_{1}, \Delta \lambda_{2}\right)$ were obtained applying the equation (1). To improve the training efficiency the data was pre-processed. Inputs and targets were normalized to have zero mean and unity standard deviation to guarantee that they have the same relevance.

The generalization ability of the trained MLP network was then verified with a set of another 100 pairs of simulated data.

\section{RESULTS}

Two fibre Bragg gratings were embedded between layers of pre-impregnated carbon fibre/epoxy resin to produce a smart laminated composite. One of the Bragg gratings is located between two layers. The other Bragg grating is positioned between four layers. The smart composite was characterized concerning stain and temperature. The characterization consisted in temperature measurements holding strain constant $(\varepsilon=0)$ and strain measurements induced by strain holding the temperature constant $\left(\mathrm{T}=20^{\circ} \mathrm{C}\right)$.

The sensing head presents different strain sensitivities of the Bragg grating when embedded in different number of layers and when the smart composite is subjected to strain. Relatively to the temperature, a nonlinear response is obtained. These results allow to write a well-conditioned system of two equations for $\Delta T$ and $\Delta \varepsilon$, given in a matrix form as: 
$\left[\begin{array}{l}\Delta \lambda_{F B G 1} \\ \Delta \lambda_{F B G 2}\end{array}\right]=\left[\begin{array}{ll}b_{1} & 0 \\ b_{2} & 0\end{array}\right]+\left[\begin{array}{cc}\kappa_{T 1} & \kappa_{\varepsilon 1} \\ \kappa_{T 2} & \kappa_{\varepsilon 2}\end{array}\right]\left[\begin{array}{l}\Delta T \\ \Delta \varepsilon\end{array}\right]+\left[\begin{array}{ll}a_{1} & 0 \\ a_{2} & 0\end{array}\right]\left[\begin{array}{c}\Delta T^{2} \\ \Delta \varepsilon^{2}\end{array}\right]$

and the solution obtained for $\Delta T$ and $\Delta \varepsilon$ is:

$\Delta T=\frac{-\left(\kappa_{T 2}-\kappa_{T 1} \frac{\kappa_{\varepsilon 2}}{\kappa_{\varepsilon 1}}\right) \pm \sqrt{\left(\kappa_{T 2}-\kappa_{T 1} \frac{\kappa_{\varepsilon 2}}{\kappa_{\varepsilon 1}}\right)^{2}-4\left(a_{2_{1}}-a_{1} \frac{\kappa_{\varepsilon 2}}{\kappa_{\varepsilon 1}}\right)\left(\frac{\kappa_{\varepsilon 2}}{\kappa_{\varepsilon 1}}\left(\Delta \lambda_{F B G 1}-b_{1}\right)-\left(\Delta \lambda_{F B G 2}-b_{2}\right)\right)}}{2\left(a_{2}-a_{1} \frac{\kappa_{\varepsilon 2}}{\kappa_{\varepsilon 1}}\right)}$

$\left.\Delta \varepsilon=\frac{1}{\kappa_{\varepsilon 1}} \backslash \lambda_{F B G 1}-\kappa_{T 1} \Delta T-a_{1} \Delta T^{2}\right]$

Replacing all the coefficient parameters, we obtained the following results:

$$
\left[\begin{array}{l}
\Delta \lambda_{F B G 1} \\
\Delta \lambda_{F B G 2}
\end{array}\right]=\left[\begin{array}{ll}
4.63 & 0 \\
3.34 & 0
\end{array}\right]+\left[\begin{array}{ll}
1.74 & 1.14 \\
1.59 & 0.55
\end{array}\right]\left[\begin{array}{c}
\Delta T \\
\Delta \varepsilon
\end{array}\right]+\left[\begin{array}{cc}
0.14 & 0 \\
0.13 & 0
\end{array}\right]\left[\begin{array}{c}
\Delta T^{2} \\
\Delta \varepsilon^{2}
\end{array}\right]
$$

and the solution is:

$$
\begin{aligned}
& \Delta T=\frac{-0.755 \pm \sqrt{+0.24 \Delta \lambda_{F B G 2}-0.11 \Delta \lambda_{F B G 1}-0.31}}{0.12} \\
& \Delta \varepsilon=0.87 \Delta \lambda_{F B G 1}-1.51 \Delta T-4.03 \Delta T^{2}
\end{aligned}
$$

The system performance was evaluated when the sensing head was simultaneously subjected to strain and temperature changes over strain and temperature ranges of 2700 $\mu \varepsilon$ and $45^{\circ} \mathrm{C}$, respectively. The results are shown in Fig. 3. The rms deviations were found to be $\pm 1.47^{\circ} \mathrm{C}$ and $\pm 5.7 \mu \varepsilon$ for temperature and strain measurements, respectively.

Usually the matrix method presents good performance when the sensing head shows linear response in all physical parameter characterization. In our design, the sensing head presents non linear response due to the optical fibre being embedded in laminated composite and presents a thermal differential between the optical glass and the polymer used in the pre-impregnated. In these cases alternative solutions are required and this work presents the results using ANN.

The performance of the two techniques (matrix method and ANN) was evaluated when the sensing head undertook strain variations in a range of $2700 \mu \varepsilon$ at a fixed temperature $\left(\Delta T=20^{\circ} \mathrm{C}\right)$ and the other way around, i.e., temperature variations in a range of $50{ }^{\circ} \mathrm{C}$ for a specific applied strain $(\Delta \varepsilon=1250 \mu \varepsilon)$. See Figure 4 . For the ANN method a satisfactorily generalization of data was achieved. The resulting maximum errors for the 
ANN method were found to be $\pm 0.2{ }^{\circ} \mathrm{C}$ and $\pm 1.9 \mu \varepsilon$ for temperature and strain measurements, respectively.

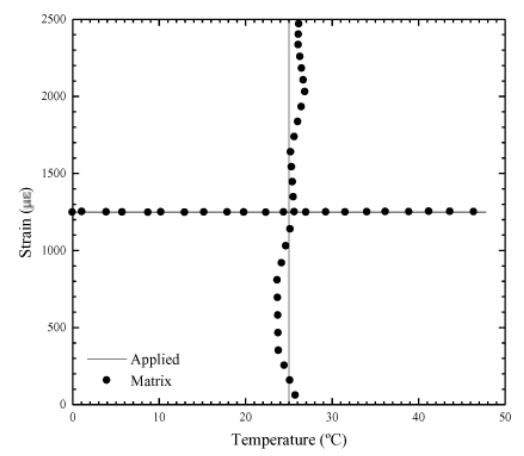

Fig. 3. Sensor output as determined by matrix method.

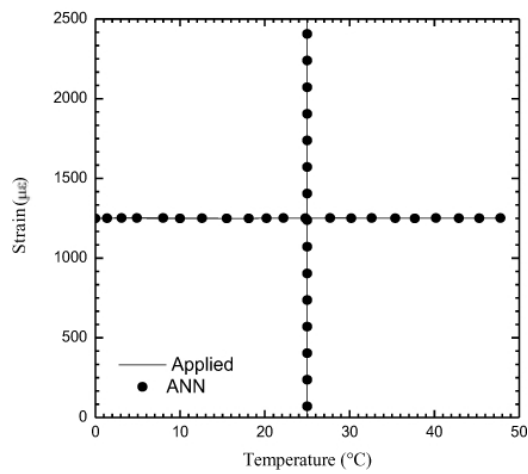

Fig. 4. Sensor output as determined by ANN.

\section{CONCLUSIONS}

In this work, the authors presented an intelligent composite based on optical Bragg grating structure embedded between of layers of pre-impregnated carbon fibre/epoxy resin. Due to its geometry, the smart composite can be simultaneously discriminate the strain and the temperature. To reduce the errors of the matrix methods, an artificial neural network (ANN) approach is proposed to improve the strain and temperature measurements crosssensitivity. The authors concluded that the ANN approach is an alternative method when the optical sensor presents non linear response to the physical parameters. The problem of the sensing head embedded in composite laminated is the non linear temperature response and the matrix method presents a high error for the temperature measurement. The results show that, for a particular sensor with large cross-sensitivity, temperature and strain measurement accuracy can be increased by 7 and 3 times, respectively, when compared with the matrix inversion method. 


\section{REFERENCES}

1. Culshaw, B. Smart structures and Materials, Artech House Publichers, 1996.

2. Chan, CC.; Jin, W., Rad, A.B., Demokan, M.S. "Simultaneous measurement of temperature and strain: an artificial neural network approach". IEEE Photonics Technology Letters, 1998; 10: 854 - 856.

3. Haykin, S. Neural Networks: a comprehensive foundation - Chapter 4: Multilayer Perceptrons, Prentice Hall, 156, 1999.

4. Bishop, C. "Neural Networks for Pattern Recognition" - Chapter 4: The Multi-layer Perceptron, Oxford: University Press, 116, 1995.

5. Demuth H., Beale M., "Neural Network Toolbox User's Guide”, Version 4. 